\title{
CHARACTERIZATION OF MOVING DUST PARTICLES
}

\author{
Brent J. Bos, Scott R. Antonille, and Nargess Memarsadeghi
}

NASA Goddard Space Flight Center, Greenbelt, Maryland, 20771.

\begin{abstract}
A large depth-of-field Particle Image Velocimeter (PIV) has been developed at NASA GSFC to characterize dynamic dust environments on planetary surfaces. This instrument detects and senses lofted dust particles. We have been developing an autonomous image analysis algorithm architecture for the PIV instrument to greatly reduce the amount of data that it has to store and downlink. The algorithm analyzes PIV images and reduces the image information down to only the particle measurement data we are interested in receiving on the ground typically reducing the amount of data to be handled by more than two orders of magnitude. We give a general description of PIV algorithms and describe only the algorithm for estimating the velocity of the traveling particles.
\end{abstract}

Index Terms - Planetary lander, velocimetry, compression, filtering, deconvolution.

\section{INTRODUCTION}

A large depth-of-field Particle Image Velocimeter (PIV) was primarily developed at NASA GSFC for characterizing fluxes of wind-borne dust particles in Martian atmosphere in particular, and planetary surfaces in general [1]. This instrument could also be adapted to terrestrial use in measuring size and velocities of opaque particles carried by natural winds and industrial gases. Examples of potential terrestrial applications include monitoring of airborne industrial pollutants and airborne particles in mine shafts. This instrument detects lofted dust particles and senses the number of particles per unit volume, measures their sizes, velocities (both speed and direction) and shape factors when the particles are large. To measure these particle characteristics in-flight, the instrument gathers two-dimensional image data at a high frame rate, typically greater than $4000 \mathrm{~Hz}$, generating large amounts of data for every second of operation $(\sim 6 \mathrm{~GB} / \mathrm{s})$. High data rates are required to sufficiently measure dynamic dust events such as dusty plumes and dust vortices. Events such as these will require several minutes of observation time by the instrument, generating more than a terabyte of data per event. Given current technology, this amount of data would be very difficult

Corresponding author: Brent 1. Bos. NASA/GSFC Code 551, Greenbelt, MD, 20771. Email: Brent.J.Bos@nasa.gov, Tel: $301-286-5670$.

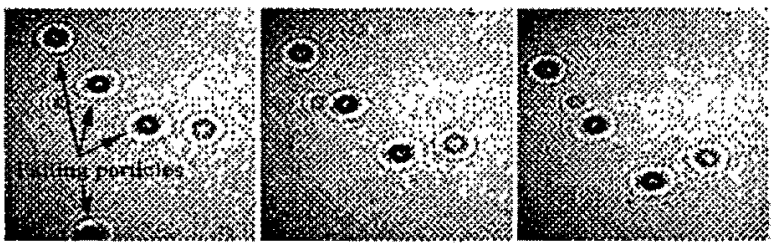

Fig. 1. PIV images of $10 \mu \mathrm{m}$ diameter particles in free-fall.

to store onboard a spacecraft and downlink to Earth. To mitigate this problem we have developed a set of image processing algorithms to dramatically reduce the amount of data that needs to be stored and downlinked. The algorithm analyzes PIV images and automatically reduces the image information down to only the particle measurement data we are interested in receiving on the ground. We have a functional algorithm architecture along with several key pieces of algorithm logic that have been proven out on 2007 and 2009 field test data acquired with a proof-of-concept PIV instrument.

The rest of this paper is organized as follows. Section 2 gives an overview of various PIV particle characterization algorithms. Then, we discuss one of these algorithms, in particular that of determining the travel direction and velocity of dust particles, in Section 3. We describe our experiments for velocity determination in Section 4. Results are presented in Section 5. Section 6 concludes the paper.

\section{PIV CHARACTERIZATION ALGORITHMS}

For small particles, approximately less than $150 \mu \mathrm{m}$ in diameter, the dust signatures that PIV captures are dominated by rotationally symmetric near-field diffraction patterns (Fig. 1). Several image processing algorithms were designed and developed for PIV in order to characterize dust particles. Characterization algorithms include counting and determining location of dust particles, their sizes, as well as direction and velocity of their movement. Fig. 3 illustrates the architecture of these algorithms. The algorithm processing begins by reading in the raw PIV images output by the instrument and then correcting the images' bad pixel signals and making the images flat fielded (Process 1). In other words, the spatial irregularities in detector characteristics and/or illumination nonuniformities are compensated for. This is a conventional im- 
age processing step typically applied to planetary image data. Next, the images are filtered (Process 2) to remove the signals of any particles that have become stuck to the PIV optics and to decrease the noise from individual pixels. This piece of algorithm logic is a novel algorithm that significantly improves the instrument's detection of small particles. We have named it the Temporal Habituation Filter (THF) since it habituates to the background noise and isolates the dust signatures. After processing images with the THF, previously undetectable dust signatures can be detected in the images. Due to the complexity and significance of this new image filter, it will be described in detail in a separate paper.

After the PIV images have been filtered, they are used as direct inputs into three separate processes. Process 3 analyzes the images to determine which particle signatures are not approximately rotationally symmetric. Those signatures are then removed from the images and processed using existing image segmentation techniques (Process 4b). But if the signatures are found to be approximately rotationally symmetric, a radial profile fitting routine (Process $4 a$ ) analyzes the signatures to determine the signature center and calculate $a$ best fit to the diffraction pattern. Processes $4 a-b$ generate the first actual particle data that we are interested in: particle positions and the number of particles per frame. Once the particle identification is completed, the particle location data and the filtered image data are input to Processes 5a-b.

Process 5b determines the particle size. For large particles, whose signatures are not dominated by near-field diffraction, their size and shape can be analyzed using conventional geometric analysis approaches. We have devoted a considerable amount of effort to develop efficient logic to determine particle sizes based on the diffraction patterns of small particles - since this is an area without much prior art. We have investigated several fast but noise-resistant particle size metrics. A statistical metric that we have found to be useful is one that includes the standard deviation of the image intensity values. This metric can be normalized in various ways but it always relates to particle diameter with a monotonic function (Fig. 2). We have also investigated metrics that measure the number and locations of the diffraction pattern oscillation points and developed an understanding of how to relate those to particle size. Of particular note is a result that we have obtained using the Hilbert-Huang Transform (HHT) [2]. Our results indicate that there is a relationship between particle size and the number of zero crossings from one of the HHT intrinsic mode functions generated by particle signatures. The most physically meaningful particle size determination logic we have developed uses conversation of energy. The images and diffraction patterns that the PIV instrument generates are shadows caused by dust particles when the particles pass in between the collimated PIV light source and the detector collection optics. So, the amount of light that is blocked from reaching the instrument's detector plane is related to the particle size. The final piece of particle information that needs to

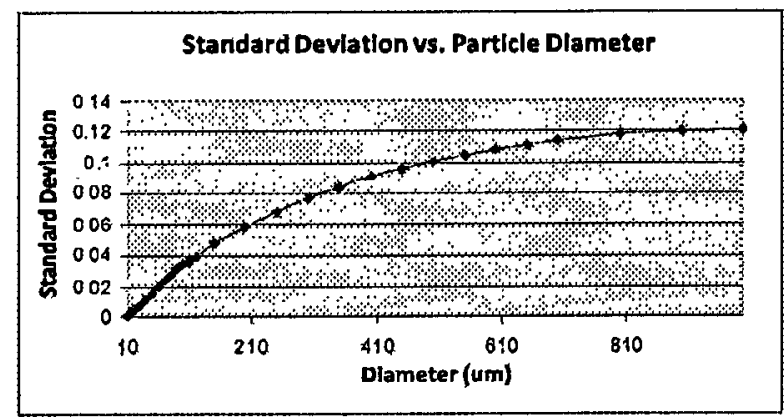

Fig. 2. Standard Deviation vs. Simulated Particle's Diameter

be extracted from the PIV image set is particle velocity - both speed and direction of travel. This is done in two steps with Process 5a and 6. Process 5a is described in Section 3.

The information obtained from Process 5a is used in Process 6 to efficiently and more accurately determine particle speed and resolve the $180^{\circ}$ ambiguity in the particle direction of travel, whenever possible. If the particle was moving too fast, it will only appear in a single frame. If this is determined to be the case, then the algorithm will not search for a signa: ture match in the next frame. If Process 6 determines that a particle could be present in the next frame, then it only looks for a signature match in those particular areas consistent with the previously determined speed and line of travel while also considering the accompanying uncertainty. This is an important piece of the Process 6 logic for small particles because as the particles get smaller, their signatures start to look more and more alike. Once particle signatures have been paired up frame-to-frame, Process 6 calculates an updated speed and travel direction for those particles that appeared in more than one frame based on the known PIV detector frame rate. For those particles that appeared in only one frame, the information generated in Process 5a is saved. The amount of data compression that PIV algorithms can achieve depends on the number of particles in each frame. For a typical scene of 20 particles per frame the amount of data is reduced by a factor more than $10^{3}$.

\section{VELOCITY DETERMINATION APPROACH}

We worked on an algorithm for estimating the travel direction and velocity of each identified particle (Process 5a and 6 in Fig. 3). The logic for this algorithm depends on the fact that the particle signatures, if blurred, will produce two areas that are noticeably brighter than others (Fig. 3). Our empirical results obtained by convolving a static simulated particle pattern with a blur function confirms this. We have also observed that elongated camera exposure times result in blurred shadow patterns of the entrained particles while the shortest exposures show nearly symmetric diffraction shadows. This indicates that the extended shadow from the entrained parti- 


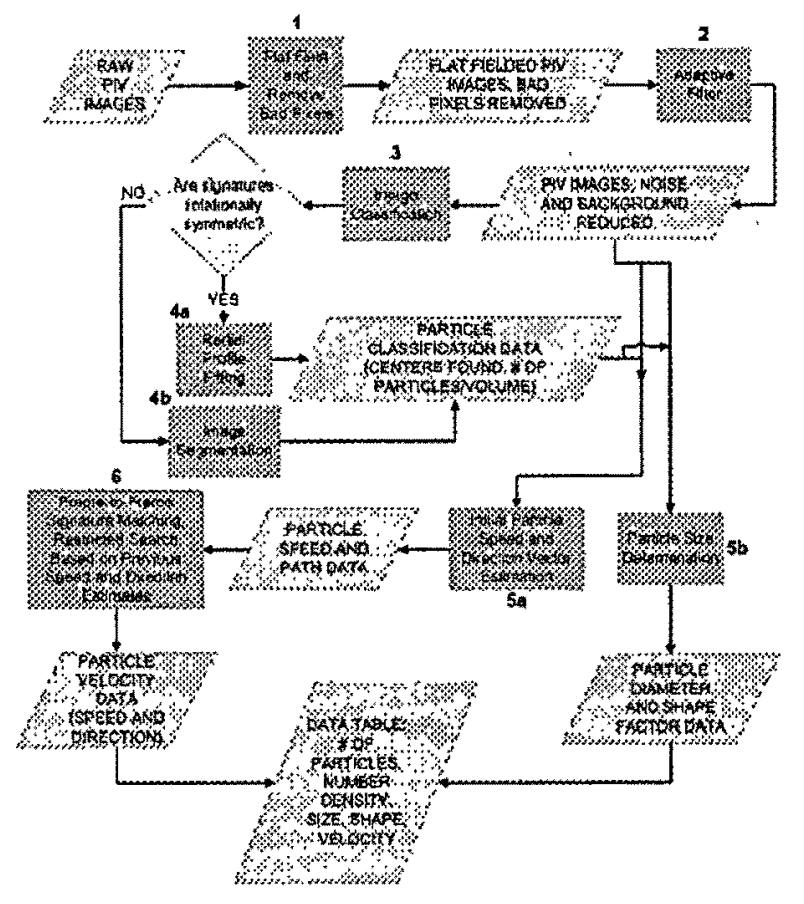

Fig. 3. PIV Data Compression Algorithm Architecture

cles is most likely from movement, and not due to their shape or material properties. Additionally, because the average effect of the particles is to lower the amount of energy that reaches the corresponding shadowed area on the detector, the particles' motion must have followed the direction in which the shadow is extended. Finally, the extent of the blurring effect is known to be consistent with both the entrained particle velocity (using anemometers) and the camera exposure time.

Process 5a takes advantage of the mentioned image smear in a single frame. Even though the PIV operates with very short detector exposure times, typically $20 \mu \mathrm{s}$, fast moving particles will still produce a blurred signature that can be measured. Fig. 3 shows a PIV dust diffraction signature for a $100 \mu \mathrm{m}$ diameter particle traveling in the direction shown by the semi-transparent arrow. There are two bright points on opposite sides of the center of the signature. A line orthogonal to the line that connects the two brightest areas will be parallel to the direction of travel. So Process 5a determines the particle direction of travel by locating the centroid of the two brightest areas on either side of the signature center, and calculating the orthogonal slope to the line that connects these two centroids. We calculated these centroids by averaging top ten brightest points in these two brightest areas. This calculation cannot determine if the particle is moving in the positive or negative direction along the line.

After the path of motion is determined, Process 5a also estimates how fast the particle was traveling. It does this by generating a series of blur kernels consisting of a single line of various lengths with a slope that is equal to the direction of the travel slope. These kernels are iteratively convolved with ideal signatures consistent with the particle size and shape, which were previously determined, until maximum agreement is achieved between the convolution and the measured signature. The known PIV exposure time can then be used with the PIV sample volume pixel scale factor to es. timate the particle speed. In other words, the initial clear dust particle image $I$ has been blurred by a filter image $B$ resulting in a blurry image of a particle similar to that of Fig. 3.

$$
B I=I * B
$$

, where * represents the convolution operator, and $B$ is a matrix of the same size as $I$ whose elements are either black or white along a line (travel vector). While the direction of the blur (the filter in image $B$ ) is perpendicular to the line connecting the two brightest areas in $B I$, its length which is a function of the particle's exposure time within the frame should be determined by solving for the blur filter that best fits Eq. 1. Since we have already determined the direction of the velocity vector, we only have to solve for its length. We expect the length of this vector to be short based on our knowledge of the frame rates and typical particle velocities. Thus, we perform an exhaustive search among several filter lengths along the estimated direction, and find the best match. Eq. 1 and the convolution theorem [3] imply

$$
\begin{aligned}
\operatorname{FFT}(B I) & =\operatorname{FFT}(I) \times \operatorname{FFT}(B) \\
\operatorname{FFT}(I) & =\operatorname{FFT}(B I) / \operatorname{FFT}(B) \\
I^{\prime} & =\operatorname{IFFT}(\operatorname{FFT}(I)),
\end{aligned}
$$

where $x$ and / represent the Schur product and division accordingly, and $I^{\prime}$ is the estimated clear image. We search for the blur function $B$ that minimizes $\left\|I-I^{\prime}\right\|$. This is possible for simulated data, for which the initial $I$ is known. For real data, we only have access to blurred image $B I$. We instead benefit from the particle size estimation provided via Process $5 b$. We then use the simulated clear image for that particle size as the clear $I$ image and repeat the same process for finding the blur image $B$.

\section{EXPERIMENTS}

We performed our velocity determination experiments on simulated data that were generated by a commercially available optical ray tracing and analysis code [4]. We designed two sets of experiments: one on clear simulated dust particles, and the other on simulated data in presence of noise. For clear images, we first generated a set of horizontal filters of lengths equal to $3,5,7,9$ and 11 pixels located in the middle of the image frames. We then rotated the filter from $0^{\circ}$ to $360^{\circ}$ in $5^{\circ}$ intervals. Finally, we convolved the clear image with the filter to create a blurry image for each case. That is, we generated 365 blurred images. 


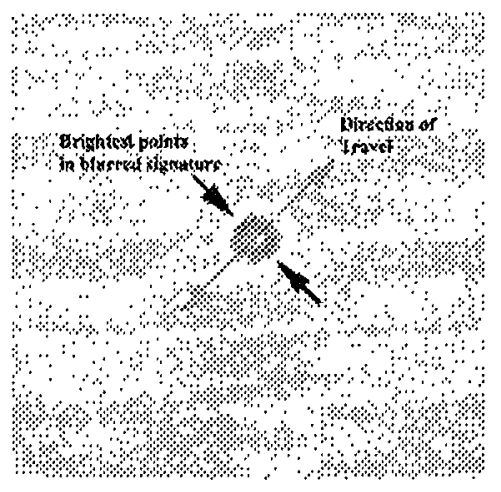

Fig. 4. Blurred $100 \mu m$ particle signature.

For noisy images, we repeated the experiments for filters of length 7 and 9 pixels only, and rotated them from $0^{\circ}$ to $180^{\circ}$ in $10^{\circ}$ intervals to obtain initial blurred images $B I_{0}$. Then, the final blurred and noisy images $B I_{n}$ were generated:

$$
B I_{n}=B I_{0}+s \times N_{i} \times B I_{0}
$$

where $s \in\{[0: 0.01: 0.1],[0.2: 0.1: 2],[2.5: 0.5: 5]\}$, and for $i \in\{1 \ldots 10\}, N_{i}$ is a random white noise image whose values are within $[-0.5,0.5]$ and have been smoothed by averaging values over $9 \times 9$ boxes. We then estimated the direction and length of the velocity vectors of these blurred images via algorithms described in Section 3.

\section{RESULTS}

Our extensive experiments on simulated data indicate that as the exposure time (length of the blurring filter) increases, our algorithm makes better direction estimations for traveling particles. Tab. 1 reports maximum and mean absolute error values of these results in degrees for simulated data in absence of noise. For each filter length, the algorithm was run 73 times for filters along $0^{\circ}: 5^{\circ}: 360^{\circ}$. While the mean absolute error for filter of length 3 is $\sim 10^{\circ}$, this error is less than $4^{\circ}$ in all other cases. Also, for $73 \%$ of all cases, we estimated the exact filter length. Our velocity determination algorithms are sen-

Table 1. Direction estimation for simulated particles traveling along $0^{\circ}$ to $360^{\circ}$ angles, with $5^{\circ}$ increments

\begin{tabular}{|c|c|c|}
\hline Filter & \multicolumn{2}{|c|}{ Absolute Error in Degrees } \\
\cline { 2 - 3 } Length & Max & Mean \\
\hline 3 & 32.57 & 9.56 \\
5 & 12.42 & 3.64 \\
7 & 08.71 & 2.73 \\
9 & 05.13 & 1.99 \\
11 & 03.73 & 1.25 \\
\hline
\end{tabular}

sitive to noise. Our results for noisy simulated data indicate that when $s$ in Eq. 3 is less than or equal to 0.6, the direction is almost always estimated within $10^{\circ}$ absolute error. We examined our algorithm by using 10 different random noise images, $N_{i}$, for each scale value $s$. Fig. 5 illustrates how for a simulated particle traveling in $40^{\circ}$ direction, increasing the noise affects the estimation error.

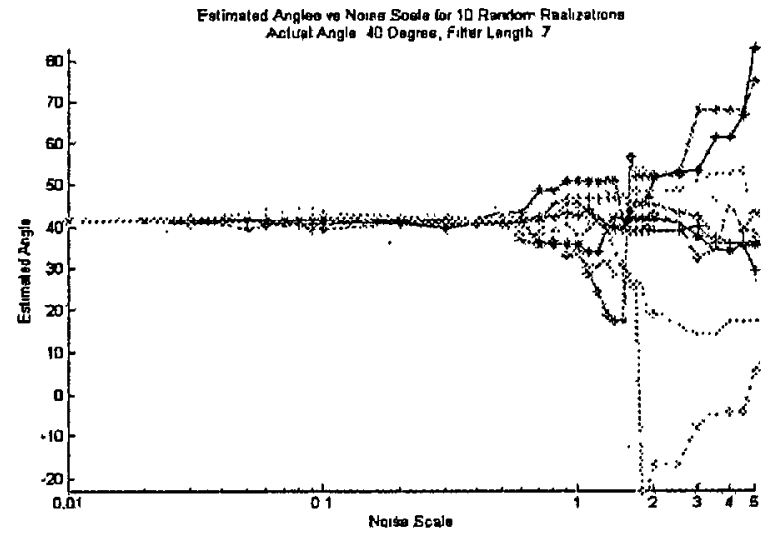

Fig. 5. Direction estimation for noisy simulated particles (travel angle: $40^{\circ}$ ).

\section{CONCLUSION}

We presented particle characterization algorithms that determine the location, size, and velocity of traveling particles via image analysis. We described the particle velocity determination algorithms. Our experiments on simulated particles imply an average absolute error of less than $4^{\circ}$ for direction estimation of traveling particles when the blurring filter length was greater than 3 pixels. Our algorithms performed well for simulated data in presence of small amounts of noise.

\section{ACKNOWLEDGMENTS}

We thank Catherine Walker and Semion Kizhner for their work on particle size determination algorithms.

\section{REFERENCES}

[1] B. J. Bos and W. M. Farrell, "Dust flux instrumentation for mars landers," in 7th International Conference on Mars, Pasadena, CA, July 2007.

[2] N. E. Haung and S. S. P. Shen, Eds., Hilbert-Haung Transform and Its Applications, ser. Interdisciplinary Mathematical Sciences. World Scientific Publishing Company, Sep. 2005, vol. 5.

[3] R. Bracewell, The Fourier Transform and Its Applications, 3rd ed. NY: McGraw-Hill, June 1999.

[4] R. Fischer, Optical System Design, 2nd ed. McGrawHill, Jan. 2008. 